\section{The age-stage issue in conservation acquisition}

\author{
CHARLES J. BRAINERD
}

University of Alberta, Edmonton, Alta., Canada T6G 2E9

The question of whether experimentally induced conservation acquisition is a function of chronological age, developmental stage, or both was examined. A total of 88 nonconserving kindergarten Ss were assigned randomly to two training conditions and two control conditions. The $44 \mathrm{Ss}$ in the two training conditions were trained on number conservation via a simple reinforcement procedure and posttested 1 week after training. An initial correlational analysis indicated that both age and stage predicted posttest performance; however, a subsequent partial correlational analysis revealed that stage was the only reliable predictor of posttest performance. These findings suggest that the benefit derived from training experiences by a nonconserving $S$ depends on the S's pretraining stage but not on the S's age.

The question of whether chronological age or developmental stage should be the principal construct around which developmental data are organized is one member of a set of familiar bipolar issues that traditionally have produced differences of opinion among developmental investigators. The two classic positions run as follows:

According to the first position, the chief impetus to psychological growth is cumulative experience. Age-the standard index of cumulative experience-is the central construct in this first approach, while stages are viewed as arbitrary partitionings of the cumulative experience variable and, therefore, as "false issues" (e.g., Bijou, 1968). The alternative position is that cumulative experience must be subordinated to developmental readiness. Stage-the standard index of readiness - is the central construct in this second approach, while age is viewed as simply a rough correlate of stage (e.g., Piaget, 1971).

The age-stage issue has been at the root of more than one dispute in the mental development literature. Perhaps the classic illustration of this fact is the long-standing disagreement about whether IQ or mental age is the better index of a child's general level of mental maturity. Recently, the age-stage issue also has arisen in the conservation training portion of the mental development literature. Each of the following hypotheses has been endorsed by one or more investigators: (1) Susceptibility to conservation training is a function of the S's age; (2) susceptibility to conservation training is a function of the S's pretraining conservation stage; (3) susceptibility to conservation training is a function of both the S's age and pretraining conservation stage.

The existing conservation training literature does not permit one to decide which of the preceding three hypotheses is correct. The published data are equivocal with respect to the hypotheses for three reasons. First, the vast majority of conservation training studies have been concerned exclusively with trainability per se and not with $S$ factors that determine susceptibility to training (Brainerd \& Allen, 1971). Second, the designs of those few investigations in which the age-trainability relation was examined (e.g., Beilin, 1965) failed to control for the confounding positive correlation between age and pretraining conservation stage. Third, the designs of those few investigations in which the stage-trainability relation was examined (e.g., Inhelder \& Sinclair, 1969) also failed to control for the confounding positive correlation between age and pretraining conservation stage. For these reasons, the present experiment was conducted. Unlike previous experiments, this experiment was designed to evaluate the separate contributions (if any) of age and stage to conservation trainability.

\section{GENERAL DESIGN}

There were four basic steps in the experiment. First, a group of kindergarten Ss was pretested for number conservation via a method that assesses each of Piaget's (1952) three stages of number conservation (Stage I = total nonconservation, Stage II = prediction of conservation prior to deformation of one of the arrays, Stage III = conservation after deformation of one of the arrays). Recent research (Brainerd \& Brainerd, 1972) indicates that Ss who violate this particular three-stage sequence are extremely rare. Second, those Ss classified as being at either Stage I or Stage II of number conservation on the pretests were trained to acquire Stage III conservation via a reinforcement procedure that has been shown to induce both weight conservation (Overbeck \& Schwartz,
1970) and quantity conservation (Brainerd, 1972a). Third, the trained Ss were posttested 1 week after training. Fourth, overall and partial correlational analyses of the posttest performance data were conducted.

\section{SUBJECTS}

A total of 155 kindergarten children (79 females and 76 females) participated in the experiment. The Ss comprised the entire enrollment of two public kindergartens located in a lower-middle-class area of Edmonton. The Ss' mean age was 5 years, 7 months. The standard deviation was 7 months and the range was 4 years, 9 months to 6 years, 5 months.

\section{APPARATUS}

A bowl of circular (red) plastic chips approximately the size of pennies was employed during the pretest and posttest phases, while an identical bowl of circular (green) chips of the same size was employed during training. During each of the phases of the experiment, chips were drawn from the bowls for use in the various problems and then returned to the bowls. The Ss' responses and the E's questions were recorded on a stereo tape recorder placed out of sight below the table.

\section{PROCEDURE}

During all phases of the procedure, the $E$ and the $S$ sat across from each other at a $3 \times 4 \mathrm{ft}$ table. The first two phases of the procedure-pretest and training-occurred on the same day, while the final phase-posttestoccurred 1 week after the first two phases.

During the pretest, the $\mathrm{E}$ first withdrew 10 chips from the bowl and placed them in a row in the center of the table at intervals of approximately 1 in. The $E$ then withdrew 10 more chips and constructed a second row such that each of the chips in the second row was directly across from a chip in the first row. The $E$ then assessed two aspects of conservation: (1) prediction of conservation prior to transformation, and (2) conservation after transformation. In Assessment 1, the rows were left exactly as they had been constructed and the $E$ asked the following questions: "If I push the pennies in this row (pointing) very close together, would our two rows each have the same number of pennies? (How do you know?) If I push the pennies in this row (pointing) very close together, would one of our two rows have more pennies then? (How do you know?) If I push the pennies in this row (pointing) very close together, would one of our rows have less pennies then? (How do you know?)" In Assessment 2, the E pushed the chips in the nearest row inward until they touched each other and asked the following questions: 
Table 1

Summary Statistics for Pretest and Posttest Performance

\begin{tabular}{|c|c|c|c|c|c|}
\hline \multirow[b]{2}{*}{ Response Mode } & \multirow{2}{*}{$\begin{array}{c}\text { Total } \\
\text { Conserver } \\
\text { Group }\end{array}$} & \multicolumn{4}{|c|}{ Group } \\
\hline & & R1 & R 2 & $\mathrm{C} 1$ & $\mathrm{C} 2$ \\
\hline \multicolumn{6}{|l|}{ Pretest Judgments } \\
\hline Mean & 10.18 & 2.33 & 2.60 & 2.56 & 2.49 \\
\hline $\mathrm{SD}$ & 1.68 & 1.77 & 1.90 & 1.52 & 1.62 \\
\hline \multicolumn{6}{|c|}{ Pretest Explanations } \\
\hline Mean & 8.84 & 1.94 & 2.09 & 2.12 & 1.97 \\
\hline SD & 2.71 & 0.98 & 1.27 & 1.01 & 1.11 \\
\hline \multicolumn{6}{|l|}{ Posttest Judgments } \\
\hline Mean & - & 9.61 & 9.79 & 2.77 & 2.68 \\
\hline SD & - & 1.81 & 1.95 & 1.84 & 1.66 \\
\hline \multicolumn{6}{|c|}{ Posttest Explanations } \\
\hline Mean & - & 7.92 & 7.88 & 2.25 & 2.06 \\
\hline SD & - & 2.43 & 2.21 & 2.00 & 1.92 \\
\hline
\end{tabular}

Note-For the total conserver group, $N=67$. For the remaining four groups, $N=22$ each.

"Do our two rows each have the same number of pennies now? (How do you know?) Does one of our rows have more pennies now? (How do you know?) Does one of our rows have less pennies now? (How do you know?)" The chips were returned to the bowl, and the $\mathrm{E}$ then constructed two more identical rows of 10 chips each. The preceding two-step assessment was repeated with two modifications: First, the E's transformation involved lengthening rather than shortening one of the rows; second, all of the E's questions focused on lengthening rather than shortening.

In all, therefore, each $S$ made a total of 12 pretest judgments (six prediction judgments and six transformation judgments) and was required to explain each judgment. A previously reported criterion (Brainerd \& Brainerd, 1972) was used to classify pretest behavior: Those Ss who made at least two out of three correct prediction judgments on both the shortening and lengthening problems plus at least two out of three correct transformation judgments on both the shortening and lengthening problems were classified as Stage III conservers and did not receive training. All other Ss were deemed to be less than total conservers (Stage I or II), and they proceeded to the training phase. This conservation criterion, which makes use only of the S's pretest judgments and not their explanations, reflects the writer's earlier argument (Brainerd, $1972 b$ ) that explanation responses in Piagetian tasks provide an overly conservative estimate of the Ss' actual levels of conceptual sophistication. By this criterion, 67 Ss were classified as total conservers and 88 Ss were classified as either Stage I or Stage II conservers. The latter group was composed of roughly equivalent numbers of Stage I conservers (49 Ss) and Stage II conservers (39 Ss).

The 88 Ss who evidenced less than Stage III conservation were assigned randomly to two experimental groups (R1 and R2) and two control groups (C1 and C2), all of which proceeded immediately to the training phase. The which were virtually the same for all four groups. The Ss in R1 were trained on only the shortening version of the number problem. On Trial 1 , the $E$ first constructed two identical rows of four chips each and then pushed the nearest row inward until the chips touched. The $\mathrm{E}$ then asked the following questions: "(1) Do our two rows each have the same number of pennies now? (2) Does one of our rows have more pennies now? (3) Does one of our rows have less pennies now?" Following each response, the $\mathrm{E}$ established eye contact with the $S$ and said either "You're right" or "You're wrong," depending on whether a given judgment was correct or incorrect. The chips were returned to the bowl and the reinforcement procedure was repeated (Trial 2) with two new rows of four chips each. Trials $3,4,5$, and 6 were the same as the first two trials, except that Trials 3 and 4 involved two rows of six chips each and Trials 5 and 6 involved two rows of eight chips each. The procedure for $\mathrm{R} 2$ was the same as for $R 1$, except that the lengthening version of the number problem was trained on all six trials. The procedure for $\mathrm{C} 1$ and $\mathrm{C} 2$ was the same as for $R 1$ and $R 2$, respectively, except that reinforcement was omitted. Thus, the Ss in R1 and R2 received a total of 18 reinforcements apiece. Also, it should be noted that only judgments and not explanations were reinforced.

One week after he or she participated in the training phase, each $S$ in the four training conditions was posttested. The posttest was a verbatim repetition of the pretest.

\section{RESULTS AND DISCUSSION}

For statistical purposes, correct posttest judgments were assigned 1s and incorrect posttest judgments were training phase consisted of six trials assigned 0s. This yielded a 13-point scale (0-12) for posttest judgments. Posttest explanations were transcribed on $3 \times 5$ cards and sorted into the following categories: (1) perceptual (e.g., This row is longer than this one); (2) don't know; (3) quantitative identity (e.g., They both have 10); (4) inversion reversibility (e.g., They were the same to begin with); (5) reciprocity reversibility(e.g., This row is longer because the pennies are spread apart); (6) addition-subtraction (e.g., You didn't add any). Explanations falling in the latter four categories were deemed adequate and assigned 1s, while those falling in the first two categories were deemed inadequate and were assigned 0 s. Thus, there also was a 13-point scale for posttest expalantions. Summary statistics for the four experimental groups appear in Table 1.

Some preliminary analyses were conducted to determine whether or not reinforcement tended to induce number conservation in previously nonconserving Ss. The consistent indication was that it did: The posttest judgments of the R1 and R2 Ss were markedly superior to those of the $\mathrm{C} 1$ and $\mathrm{C} 2 \mathrm{Ss}(\mathrm{t}=17.95, \mathrm{df}=86$, $\mathrm{p}<.0001)$; the posttest explanations of the R1 and R2 Ss were markedly superior to those of the $\mathrm{C} 1$ and $\mathrm{C} 2 \mathrm{Ss}$ $(\mathrm{t}=12.41, \mathrm{df}=86, \mathrm{p}<.0001)$. Inspection of the protocols of individual $S s$ revealed that there was no overlap between the judgment performances of the reinforcement and control Ss-even the worst reinforcement $\mathrm{S}$ performed better than the best control $S$. The posttest performances of the reinforcement Ss also were compared with the pretest performances of the 67 total conservers who did not receive training. The comparisons indicated that there were only slight differences between the two groups: The judgments of the two groups did not differ significantly $(t=1.40, d f=109$, $p>.10$, in favor of the total conservers); however, the explanations of the total conservers were marginally superior to those of the reinforcement Ss $(t=1.88, \mathrm{df}=109, \mathrm{p}<.10)$.

To evaluate the extent to which age and/or pretraining stage determines the trainability of number conservation, the overall and partial correlations among three triads of variables were computed: (1) age (in months), pretraining judgments, posttest judgments; (2) age, pretraining explanations, posttest explanations; (3) age, combined pretraining judgments and explanations, combined posttest judgments and explanations. The second variable in each triad reflects pretraining stage. Only the data of the 
$R 1$ and $R 2$ Ss were employed in these analyses. The results of the overall and partial correlational analyses appear in Table 2. When the three posttest variables (judgments, explanations, combined) are considered as performance criteria which the age and stage variables should predict, a clear trend is apparent: While each of the overall correlations between posttest performance and age and between posttest performance and stage are significant, the only partial correlations that are significant are those between posttest performance and pretraining stage. When the age-posttest correlations are corrected for the linear regression of age on pretraining stage, the resultant coefficients remain significant.

Thus, the present findings are consistent with Hypothesis 2 mentioned in the introduction to this paper. Moreover, the findings permit this hypothesis to be carried a step further: The trainability of the present Ss depended on their pretraining stage exclusive of their age. These findings also suggest the possibility that previously reported evidence in support of an age-trainability correlation may well be an artifact of the confounding correlation between age and pretraining stage.

In contrast with these conclusions, it perhaps could be maintained that a

Table 2

Overall and Partial Correlations Between Age, Pretraining Stage, and Posttest Performance

\begin{tabular}{|c|c|c|c|c|}
\hline \multirow[b]{2}{*}{ Response Mode } & \multicolumn{2}{|c|}{ Overall Correlations } & \multicolumn{2}{|c|}{ Partial Correlations } \\
\hline & Age & Stage & Age & Stage \\
\hline Posttest Judgments & $.44^{* *}$ & $.69 \dagger$ & .13 & $.60 \dagger$ \\
\hline Posttest Explanations & $.29^{*}$ & $.63 \dagger$ & -.02 & $.59+$ \\
\hline Posttest Combined & $.48 * *$ & $.78 \dagger$ & .08 & $.70 \dagger$ \\
\hline
\end{tabular}

positive partial correlation between age and trainability might have emerged if the age range of the $S$ sample had been broader. This is a very remote possibility, however. The age range of the present $S$ sample includes the entire interval during which number conservation is known to develop in North American children. Sampling from a broader age range therefore should not tend to increase the age-trainability correlation.

\section{REFERENCES}

BEILIN, H. Learning and operational convergence in logial thought development. Journal of Experimental Child Psychology, 1965, 2, 317-339.

BIJOU, S. W. Ages, stages and the naturalization of human development. American Psychologist, 1968, 23 . 419-427.

BRAINERD, C. J. Reinforcement and reversibility in quantity conservation acquisition. Psychonomic Science, 1972a, 27, 114-116.
BRAINERD C J Judgments and explanations as criteria for the presence of cognitive structures. Psychological Bulletin, $1972 \mathrm{~b}$, in press.

BRAINER D, C. J., \& ALLEN, T. W. Experimental inductions of the conservation of "first-order" quantitative invariants. Psychological Bulletin, 1971, 75, 128-144.

BRAINERD, C. J., \& BRAINERD, S. C. Order of acquisition of number and liquid quantity conservation. Child Development, 1972, 43, in press.

INHELDER, B, \& SINCLAIR, H. Learning cognitive structures. In $\mathrm{P} . \mathrm{H}$. Mussen, J. Langer, and M. Covington (Eds.), Trends and issues in developmental psychology. New York: Holt, Rinehart \& Winston, 1969

OVERBECK, C., \& SCHWARTZ, M. Training in conservation of weight. Journal of Experimental Child Psychology, 1970, 9, 253-264.

PIAGET, J. The child's conception of number. New York: Humanities, 1952.

PIAGET, J. The theory of stages in cognitive development. In D. R. Green, M. P. Ford, and G. B. Flamer (Eds.), Measurement and Piaget. New York: McGraw-Hill, 1971. 